\title{
Household food insecurity and children's physical activity and sedentary behaviour in the United States: the Healthy Communities Study
}

\author{
Sophia M Navarro ${ }^{1}$ (D), Marisa M Tsai ${ }^{1}$ (1), Lorrene D Ritchie ${ }^{1}\left(0\right.$, Edward A Frongillo $^{2}$, \\ Barbara A Laraia ${ }^{3}$, Russell R Pate ${ }^{2}$ and Lauren E Au ${ }^{4, *}$ (i) \\ 'Nutrition Policy Institute, Division of Agriculture and Natural Resources, University of California, Oakland, CA, USA: \\ ${ }^{2}$ Arnold School of Public Health, University of South Carolina, Columbia, SC, USA: ${ }^{3}$ School of Public Health, University \\ of California, Berkeley, Berkeley, CA, USA: ${ }^{4}$ Department of Nutrition, University of California, Davis, 3215 Meyer \\ Hall, One Shields Avenue, Davis, CA 95616, USA
}

Submitted 11 December 2020: Final revision received 13 May 2021: Accepted 4 June 2021: First published online 10 June 2021

\begin{abstract}
Objective: To examine associations between household food insecurity and children's physical activity and sedentary behaviours.

Design: Secondary analysis was conducted on the Healthy Communities Study, an observational study from 2013 to 2015 . Household food insecurity was assessed by two items from the US Department of Agriculture's 18-item US Household Food Security Survey Module. Physical activity was measured using the 7-d Physical Activity Behaviour Recall instrument. Data were analysed using multilevel statistical modelling.

Setting: A total of 130 communities in the USA.

Participants: In sum, 5138 US children aged 4-15 years.

Results: No associations were found for the relationship between household food insecurity and child physical activity. A significant interaction between household food insecurity and child sex for sedentary behaviours was observed $(P=0.03)$. Conclusions: Additional research capturing a more detailed assessment of children's experiences of food insecurity in relation to physical activity is warranted. Future studies may consider adopting qualitative study designs or utilising food insecurity measures that specifically target child-level food insecurity. Subsequent research may also seek to further explore sub-group analyses by sex.
\end{abstract}

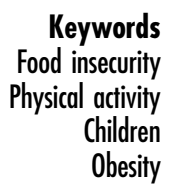

In 2019, 13.7 million households in the USA experienced food insecurity ${ }^{(1)}$. Among households with children, 2.4 million had children who were food insecure ${ }^{(1)}$. High prevalence of food insecurity was observed in households with children (13.6\%) and those headed by single mothers or single fathers (28.7\% and $15.4 \%$, respectively). Additionally, non-Hispanic Black households, Hispanic households and low-income households below 185\% of the poverty line were also observed to have high prevalence of food insecurity $(19 \cdot 1 \%, 15 \cdot 6 \%$ and $27 \cdot 6 \%$, respectively $)^{(1)}$. The prevalence of household food insecurity was highest for those living in the Southern USA (11.2\%) and in the principal cities of metropolitan areas $(12.4 \%)^{(1)}$.

Food insecurity is associated with poorer physical health, cognitive development, academic achievement and behaviour in children and has been linked to higher odds of childhood obesity ${ }^{(2-4)}$. Risk of childhood obesity is also associated with physical activity and sedentary behaviours ${ }^{(5,6)}$. Few studies have investigated the connections between food insecurity and physical activity in children, however, and those that have explored this link demonstrate mixed associations, with some finding negative associations with moderate-to-vigorous physical activity, null or negative associations with sedentary behaviour and null associations with total physical activity, defined as the sum of light, moderate and vigorous intensity physical activity $^{(7-9)}$. It is hypothesised that food insecure children may be less likely to participate in physical activity because food insecurity can have physical (e.g. hunger, fatigue and illness) and emotional (e.g. stress, anxiety and depression) manifestations ${ }^{(10,11)}$. Thus, because both food insecurity and inadequate physical activity can result in serious health

*Corresponding author: Email leau@ucdavis.edu

C The Author(s), 2021. Published by Cambridge University Press on behalf of The Nutrition Society. This is an Open Access article, distributed under the terms of the Creative Commons Attribution licence (http://creativecommons.org/licenses/by/4.0/), which permits unrestricted re-use, distribution, and reproduction in any medium, provided the original work is properly cited. 
consequences and interventions aimed at decreasing food insecurity may positively impact physical activity, it is critical to examine this relationship in children. This research will help clarify the relationship between food insecurity and physical activity, contributing meaningful insights into the implications of food insecurity in children.

The primary aim of this study was to examine whether household food insecurity is associated with children's physical activity and sedentary behaviour. Prior studies have indicated that factors influencing physical activity may operate inconsistently across certain subgroups, such as age, sex and race/ethnicity. For example, trends in physical activity behaviour among US youth reveal that children aged 12-15 years tend to be less physically active than children aged 6-11 years, high-school girls tend to be less physically active than high-school boys and there are reported differences in activity by race/ethnicity ${ }^{(12)}$. Thus, this study will also explore whether the relationship between food insecurity and physical activity differs within specific subpopulations, including age, sex and, race/ ethnicity.

\section{Methods}

\section{Study design and participants}

The Healthy Communities Study is a cross-sectional observational study of 5138 US children designed to assess the impact of community programmes and policies addressing childhood obesity on child diet, physical activity and weight outcomes. Participants included those 4-15 years in grades $\mathrm{K}-8$ and were chosen from a diverse sample of 130 communities, defined as high-school catchment areas, across the country between 2013 and 2015. Community selection was based on hybrid sampling that included a national probability-based sample of 102 communities as well as a sample of twenty-eight 'certainty' communities known for having programmes and policies that target childhood obesity prevention. Data collectors interviewed children and families in their homes, gathering comprehensive information on demographics, nutritional status, anthropometry, physical activity and the surrounding environment. The Battelle Memorial Institute Institutional Review Board approved the study protocol, and parents provided written informed consent for child participation. A more detailed explanation of participant recruitment, study design and data collection are included in papers by Sagatov et al., Strass et al. and John et al. ${ }^{(13-15)}$.

\section{Variables of interest}

Demographic information was provided by the parent or guardian and included items on child age, child race/ ethnicity, geographic region, urbanicity, country of origin, household income, parental education attainment, marital status and employment status. Household food insecurity was indicated by two items from the US Department of Agriculture's 18-item US Household Food Security Survey Module that assess uncertainty that the household food budget or food supply is sufficient to meet basic needs ${ }^{(16)}$. The indicator from these two items has high sensitivity (97\%) and specificity (83\%) in predicting responses to the full module and are associated with poor or fair child health, hospitalisations and developmental risk ${ }^{(17)}$. Furthermore, any affirmation of household food insecurity is associated with children's academic performance, weight gain and social skills ${ }^{(18)}$. Administered by an interviewer, respondents were asked to assess how often the following occurred within the past 12 months: (1) 'We were worried whether our food would run out before we got money to buy more' and (2) 'The food we bought just didn't last and we didn't have money to get more.' Participants could respond with very often, often, sometimes, rarely or never. Those who responded with very often, often or sometimes for at least one of the items were categorised as having household food insecurity. For all households, the adult was the respondent.

Physical activity was measured using self-report in the 7-d Physical Activity Behavior Recall (PABR-7) instrument. The tool was designed to detect forms of physical activity and sedentary behaviour that are typically targeted in community interventions to reduce childhood obesity. Through a computer-assisted interview, respondents specified whether the child participated in each activity over the past $7 \mathrm{~d}$, the days the activity was performed and the average intensity of the activity (i.e. light, moderate, hard and very hard) using sex- and age-appropriate visual aids. For children aged 4-8 years, their parent/guardian responded, and the child was there to assist. For children aged 9-15 years, the child responded, and their parent/guardian was present to assist ${ }^{(19)}$. In this study, physical activity terms refer to the frequency and intensity of specific forms of activity and are therefore used in a manner that is specific to the methodology of the study.

Four measures were used to assess a child's physical activity: total physical activity, vigorous physical activity, moderate-to-vigorous physical activity and sedentary behaviours. These variables were converted into index scores by multiplying the number of reported activities for each activity intensity level by the frequency of participation in those activities. Greater scores indicate more physical activity and sedentary behaviour. The total physical activity index score included fifteen activities: PE class, recess/free play, dance, activity breaks, school sports, non-school sports, pick-up sports, physical activity in after-school, physically active games, swim/water activities, outdoor/adventure activities, walk/bike to school, walk/bike to store/friend's house/etc., walk/bike for fun/ exercise and active video games. The vigorous physical activity index score included four activities: school sports, non-school sports, pick-up sports and outdoor/adventure activities. The moderate-to-vigorous physical activity index 
score included eleven activities: PE class, recess/free play, dance, school sports, non-school sports, pick-up sports, physical activity in after-school, physically active games, swim/water activities, outdoor/adventure activities and walk/bike for fun/exercise. The sedentary behaviours index score included four activities: used a computer for games or playing on the internet (not for schoolwork or social networks), used a computer or phone for social networking, watched TV and played non-active video games $^{(19)}$.

\section{Data analysis}

Secondary data analysis was conducted on the Healthy Communities Study data set. Participant demographics were analysed by household food insecurity status using t-tests for continuous variables and $\chi^{2}$ tests of independence for categorical variables. Multilevel linear regression models were generated to relate food insecurity with physical activity, adjusting for child- and community-level covariates and clustering by school and community levels. Multiple imputation by chained equations was used to account for missing data due to participant nonresponse whereby twenty imputed data sets were created and then combined $^{(20)}$. Approximately a quarter of cases $(24.5 \%)$ were missing at least one variable. The least absolute shrinkage and selection operator (LASSO) technique was used to select a subset of covariates from the large pool of demographic variables available, increasing accuracy and interpretability of models ${ }^{(21)}$. The covariates included in the models were maximum parental education, maximum parental employment status, child age, child age squared, child race/ethnicity, seasonality, US region, income classification, community percentage of African Americans, community percentage of high school graduates and community unemployment rate for those in the labour force 16 years and older.

Additional, exploratory multilevel linear regression models were generated to assess for interaction with child age, sex and race/ethnicity. Child sex was coded as female or male, based on the identification by the data collector. The race/ethnicity variable had four options: Non-Hispanic White, Non-Hispanic African American, Non-Hispanic other and Hispanic or Latino. Based on a review of the relevant literature assessing how physical activity varies by age, age was divided into 4-11 years and $12-15$ years $^{(12)}$. All analyses were conducted in SAS 9.4 (SAS Institute). Reported $P$-values are two-sided, and significance was set at $P<0.05$.

\section{Results}

In this study sample, children were on average 9 years old and half were female (Table 1). Nearly half of children were Hispanic or Latino (44.7\%). The majority (72.9\%) had at least one parent working full-time; less than half of parents $(42.9 \%)$ had a maximum education of high school or less. Geographically, $41.6 \%$ of participants were from the Southern USA. At the community level, nearly $19.7 \%$ of children lived in a community with a census tract minority classification of African American and 34.8\% with an income classification of low income $e^{(22,23)}$.

Close to half of children were classified as residing in a household with food insecurity, with the highest prevalence seen among Hispanic or Latino children (58.1 \%) followed by non-Hispanic African American children (21.5\%) (Table 1). A high prevalence of household food insecurity was also observed in children whose parents had a maximum educational attainment of some college or Associate degree or less $(87.9 \%)$. Additionally, those living in the Southern USA ( $43.7 \%$ ) had higher prevalence of household food insecurity. On the community level, communities with lower income classifications, greater proportions of African American children, higher unemployment rates and fewer high school graduates also had higher prevalence of household food insecurity.

Children on average had a total physical activity index score of 17.9 , a vigorous physical activity index score of $3 \cdot 0$, a moderate-to-vigorous physical activity index score of 13.5 and a sedentary activity index score of 11.8 (Table 1). Children in households with food insecurity had less moderate-to-vigorous physical activity than children in households without food insecurity $(P<0.001)$.

No associations were observed between household food insecurity and physical activity (Table 2). A significant interaction between food insecurity and child sex at the sedentary level was found $(P=0.03)$ (Table 3 ). When stratified to explore individual effects by sex, associations between food insecurity and physical activity were NS for either females or males (data not shown). There were no other associations by race/ethnicity or age (data not shown).

\section{Discussion}

The results from the exploratory analysis indicate that sex may modify the relationship between household food insecurity and physical activity in children. Girls in households with food insecurity engaged in more sedentary behaviours than did girls in households without food insecurity. In contrast, boys in households with food insecurity engaged in less sedentary behaviours compared with boys in households without food insecurity. Food insecurity in the USA impacts school-age girls more than boys and is associated with poorer academic performance and higher BMI ${ }^{(18,24,25)}$. Additionally, participation in the Supplemental Nutrition Assistance Program is associated with better academic performance of girls more than boys, suggesting that girls may be more susceptible to some consequences of food insecurity ${ }^{(26)}$. Since stratification by sex did not yield significant results, additional studies are needed to better 
Table 1 Socio-demographic characteristics of the children in the Healthy Communities Study by household food insecurity status ( $n$ 5138)

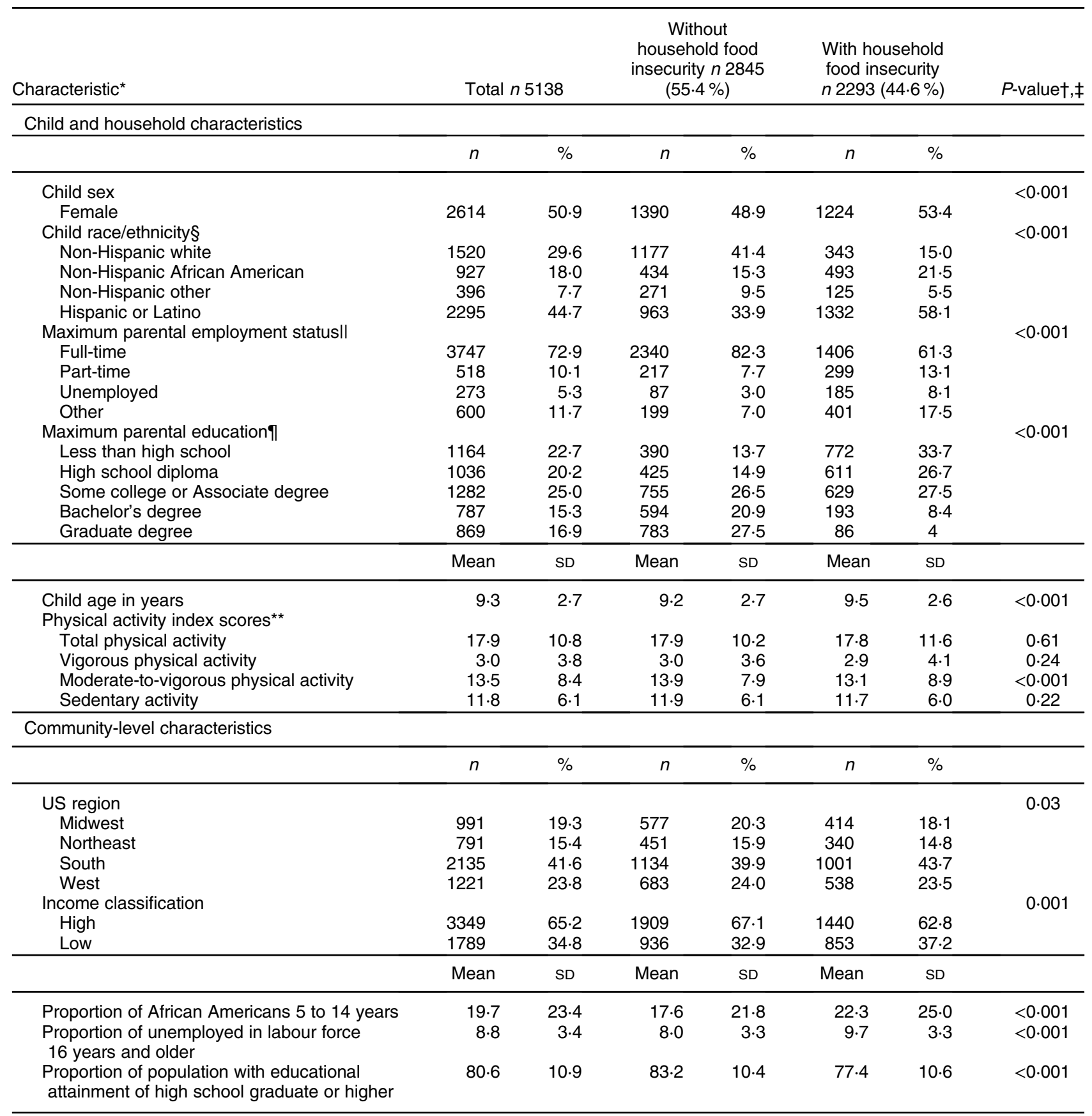

*Variables selected using the LASSO technique.

†Statistical significance was set at $P<0.05$.

†Results from $\chi^{2}$ tests of independence for categorical variables or t-tests for continuous variables. Multiple imputation by chained equations employed to account for missing values. Approximately a quarter $(24.5 \%)$ of cases were missing at least one variable.

$\S$ Child race/ethnicity 'other' category includes Asian, Native Hawaiian/Pacific Islander, Non-Hispanic American Indian/Alaska Native, more than one race including African American and more than one race not including African American.

IIUnemployed includes those unemployed and looking for work; other includes those who are only temporarily laid off, on sick leave, or maternity leave, disabled, keeping house, retired, student and other.

TGraduate degree includes Master's degree, professional degree and doctoral degree.

${ }^{\star *}$ Physical activity index scores represent the multiplication of the number of reported activities for each activity intensity level within the past $7 \mathrm{~d}$ by the frequency of participation in those activities. Greater scores indicate more physical activity and sedentary behaviours.

understand the influence of sex, physical activity and food insecurity.

One potential explanation for the lack of association in the main analysis lies with the food insecurity indicator used. In this study, household food insecurity was assessed by two items from the US Department of Agriculture's eighteen-item measure that identifies households experiencing uncertainty about food access. A previous study 
Table 2 Associations of household food insecurity and physical activity of the children in the Healthy Communities Study as measured by the 7-d Physical Activity Behaviour Recall (PABR-7) instrument $(n$ 5138)

\begin{tabular}{|c|c|c|c|}
\hline \multirow[b]{2}{*}{ Activity type } & \multicolumn{2}{|c|}{$\begin{array}{l}\text { Household food } \\
\text { insecurity }^{*}\end{array}$} & \multirow[b]{2}{*}{$P$-value $†, \ddagger$} \\
\hline & $\beta$ & $95 \% \mathrm{Cl}$ & \\
\hline $\begin{array}{l}\text { Total physical activity index } \\
\text { score§ }\end{array}$ & 0.09 & $-0.59,0.77$ & 0.79 \\
\hline $\begin{array}{l}\text { Vigorous physical activity index } \\
\text { scorell }\end{array}$ & -0.05 & $-0.30,0.19$ & 0.66 \\
\hline $\begin{array}{l}\text { Moderate-to-vigorous physical } \\
\text { activity index score }\end{array}$ & $-0 \cdot 12$ & $-0.65,0.41$ & 0.66 \\
\hline $\begin{array}{l}\text { Sedentary activity index } \\
\text { score }^{\star \star}\end{array}$ & $-0 \cdot 12$ & $-0.50,0.27$ & 0.55 \\
\hline
\end{tabular}

*Binary predictor of interest was having household food insecurity (1) compared with not having (0) household food insecurity.

tStatistical significance was set at $P<0.05$

¥Multilevel mixed modelling adjusted for maximum parental education, maximum parental employment status, child age, child age squared, child race/ethnicity, seasonality, US region, income classification, community percentage of African Americans, community percentage of high school graduates and community unemployment rate for those in the labour force 16 years and older. Standard errors are clustered at the community and school levels. Multiple imputation by chained equations employed to account for missing values. Approximately a quarter $(24.5 \%)$ of cases were missing at least one variable.

$\S$ Total physical activity index score is the number of reported activities (out of 15) multiplied by the frequency of participation in those activities in the past $7 \mathrm{~d}$.

IIVigorous physical activity index score is the number of reported activities (out of 4) multiplied by the frequency of participation in those activities in the past $7 \mathrm{~d}$.

IModerate-to-vigorous physical activity index score is the number of reported activities (out of 11) multiplied by the frequency of participation in those activities in the past $7 \mathrm{~d}$.

**Sedentary activity index score is the number of reported activities (out of 4 ) multiplied by the frequency of participation in those activities in the past $7 \mathrm{~d}$.

demonstrated that the two-item tool was sensitive, specific and valid when tested in a sample of 30098 families with young children of which $23 \%$ were food insecure ${ }^{(17)}$, but these two items may not capture the experience of all individuals in the household, particularly children. Although parents attempt to protect their children from food insecurity, children are often aware of food insecurity and take responsibility for it ${ }^{(27)}$. Children's experiences of food insecurity are often not known by parents ${ }^{(27)}$. Many children have greater awareness of household food insecurity than parents assume, and child report of their food insecurity is more strongly associated with outcomes than parent report ${ }^{(28)}$. Perhaps there is a relationship between food insecurity and physical activity in children, but the use of this two-item household food insecurity indicator was unable to detect this association by not assessing child experiences of food insecurity.

Previous studies that assessed the relationship between food insecurity and physical activity among children have also not used food insecurity measures that specifically assess children, such as the five-item questionnaire used in the US National Health and Examination Survey ${ }^{(8,29)}$ and the US Department of Agriculture's eighteen- or six-item measures, which may have contributed to the mixed findings ${ }^{(9,30)}$. Using measures that specifically assess child food insecurity may be able to more accurately capture this relationship. The US Child Food Security Survey Module is one validated child-report measure for older children aged 12 to 17 years ${ }^{(31)}$, but it is not recommended for use in younger children. Additionally, because it was adapted from selected items in the US Household Food Security Survey Module for adults, some researchers suggest that it reflects adult experiences of food insecurity that some children may not share ${ }^{(7,32,33)}$. The Child Food Security Assessment is another self-reported measure that is validated for use in children as young as 6 years of age. Unlike the US Household and Child Food Security

Table 3 Moderating effect of child sex on the association between household food insecurity and physical activity of the children in the Healthy Communities Study as measured by 7-d Physical Activity Behaviour Recall (PABR-7) instrument ( $n$ 5138)

\begin{tabular}{|c|c|c|c|c|c|c|c|c|c|}
\hline \multirow[b]{3}{*}{ Activity type } & \multicolumn{4}{|c|}{ Female $n 2614(50.9 \%)$} & \multicolumn{4}{|c|}{ Male $n 2524(49.1 \%)$} & \multirow{3}{*}{$\begin{array}{c}P \text {-value for } \\
\text { interaction } \\
\text { by } \operatorname{sex}^{*}, \dagger\end{array}$} \\
\hline & \multicolumn{2}{|c|}{$\begin{array}{c}\text { Without household } \\
\text { food insecurity } \\
n 1390(48.9 \%) \\
\end{array}$} & \multicolumn{2}{|c|}{$\begin{array}{l}\text { With household } \\
\text { food insecurity } \\
n 1224(53.4 \%)\end{array}$} & \multicolumn{2}{|c|}{$\begin{array}{c}\text { Without household } \\
\text { food insecurity } \\
n 1455(51.1 \%) \\
\end{array}$} & \multicolumn{2}{|c|}{$\begin{array}{l}\text { With household } \\
\text { food insecurity } \\
n 1069(53.4 \%)\end{array}$} & \\
\hline & $\beta$ & $95 \% \mathrm{Cl}$ & $\beta$ & $95 \% \mathrm{Cl}$ & $\beta$ & $95 \% \mathrm{Cl}$ & $\beta$ & $95 \% \mathrm{Cl}$ & \\
\hline $\begin{array}{l}\text { Total physical activity index } \\
\text { score } \neq\end{array}$ & $17 \cdot 33$ & $16 \cdot 12,18.55$ & $17 \cdot 15$ & $15 \cdot 89,18.41$ & $18 \cdot 55$ & $17 \cdot 33,19 \cdot 77$ & $19 \cdot 04$ & $17 \cdot 78,20 \cdot 29$ & 0.26 \\
\hline $\begin{array}{l}\text { Vigorous physical activity index } \\
\text { score§ }\end{array}$ & $2 \cdot 34$ & $1.93,2.75$ & $2 \cdot 44$ & $2 \cdot 01,2 \cdot 86$ & 3.52 & $3 \cdot 11,3.93$ & 3.39 & $2 \cdot 96,3 \cdot 81$ & 0.27 \\
\hline $\begin{array}{l}\text { Moderate-to-vigorous physical } \\
\text { activity index scorell }\end{array}$ & $13 \cdot 18$ & $12 \cdot 24,14 \cdot 13$ & 12.99 & $12 \cdot 01,13.97$ & $13 \cdot 90$ & $12 \cdot 95,14.85$ & $13 \cdot 91$ & $12.93,14.88$ & 0.66 \\
\hline Sedentary activity index score & 11.46 & $10 \cdot 82,12 \cdot 10$ & $11 \cdot 74$ & $11.08,12.41$ & $13 \cdot 12$ & $12 \cdot 49,13.76$ & $12 \cdot 69$ & $12 \cdot 03,13 \cdot 35$ & 0.03 \\
\hline
\end{tabular}

* Statistical significance was set at $P<0.05$.

†Analyses used multilevel modelling to identify moderating effects of sex on the association between household food insecurity with physical and sedentary activity. Models included an interaction term for food security status (with $v$. without) by child sex (female $v$. male). Covariates included maximum parental education, maximum parental employment status, child age, child age squared, child race/ethnicity, seasonality, US region, income classification, community percentage of African Americans, community percentage of high school graduates and community unemployment rate for those in the labor force 16 years and older. Standard errors are clustered at the community and school levels. Multiple imputation by chained equations employed to account for missing values. Approximately a quarter (24.5\%) of cases were missing at least one variable.

$¥$ Total physical activity index score is the number of reported activities (out of 15) multiplied by the frequency of participation in those activities in the past $7 \mathrm{~d}$ $\S$ Vigorous physical activity index score is the number of reported activities (out of 4) multiplied by the frequency of participation in those activities in the past 7 d. IIModerate-to-vigorous physical activity index score is the number of reported activities (out of 11) multiplied by the frequency of participation in those activities in the past $7 \mathrm{~d}$. ๆSedentary activity index score is the number of reported activities (out of 4 ) multiplied by the frequency of participation in those activities in the past $7 \mathrm{~d}$. 
Survey Modules, the Child Food Security Assessment was developed based on interviews with children and assesses multiple subdomains of child food insecurity including cognitive awareness, emotional awareness, physical awareness, participation in parent food strategies, initiation of child food management strategies and resource generation $^{(32)}$.

Further, given that the physical activity tool, PABR-7, was based on self-report, it may be subject to recall error. The inclusion of objective measures of physical activity, such as data collected from wearable activity tracking devices, was available but limited to $10 \%$ of the full study sample due to the original study's budgetary constraints $^{(19)}$. The tool has not yet been validated against accelerometry. Additionally, although the PABR-7 focused on activities targeted by community programmes and policies, which could exclude other important forms of physical activity, it was structured similar to other physical activity self-report instruments that have been widely used in previous studies with children ${ }^{(19)}$. The tool reflects the composite of the child's participation in multiple types of physical activity that are often offered in communities that are implementing obesity prevention programmes for children and youth. Therefore, the instrument and composite index should be reflective of the child's engagement in activities that are often conceptualised as supporting prevention of obesity.

The Healthy Communities Study used a large, diverse sample of children and households from across the United States ${ }^{(1)}$ with a high prevalence of household food insecurity (44.6\%) and childhood obesity $(24.8 \%)^{(34)}$ compared with national estimates of food insecurity in households with children $\left(19.5 \%{ }^{(35)}\right.$ and $\left.16.6 \%{ }^{(36)}\right)$ and childhood obesity $(17 \cdot 2 \% \text { and } 18.5 \%)^{(37)}$ in 2013 and 2015 , respectively. The study oversampled communities with certain criteria, including high proportions of African American, Hispanic and low-income households, to lend statistical power and relevance to populations experiencing health disparities ${ }^{(38)}$. While the study was conducted between 2013 and 2015 and has not been repeated since, the findings are relevant to today's public health context as trends in food insecurity and childhood obesity in the United States remain high, especially in low-income and racially diverse populations. The coronavirus pandemic has increased the prevalence of child food insecurity to a projected $17.9 \%$ in $2021^{(39)}$, and recent data suggest that food insecurity in households with children is close to a combined $40.0 \%$ among African American and Hispanic households and $37 \cdot 1 \%$ among households with an income-to-poverty ratio under $1 \cdot 0^{(40)}$. Additionally, childhood obesity has risen in 2017-2018 to $19.3 \%$ and is estimated to be a combined $49.8 \%$ among African American and Hispanic children ${ }^{(37)}$ and close to $38.8 \%$ in households with incomes less than or equal to $350 \%$ of the federal poverty level ${ }^{(41)}$. Given its observational design, the study is unable to determine causal relationships.

\section{Conclusion}

This study suggests that sex may modify the relationship between household food insecurity and physical activity in children, though additional research is needed to clarify this relationship. Results from this study indicate that use of a more detailed food insecurity measure that better represents child experience may be warranted to assess how severity of child food insecurity is contemporaneously related to physical activity. Future studies may also explore more sub-group analyses by sex or design qualitative studies to better understand this relationship from the perspective of children.

\section{Acknowledgements}

Acknowledgements: The authors would like to acknowledge Kaela Plank and Sridharshi Hewawitharana for their assistance with data analysis and Laurel Moffat and Isabel Thompson for their feedback in manuscript development. Financial support: Research reported in this publication was supported by the National Heart, Lung, And Blood Institute of the National Institutes of Health under Award Number K01HL131630. The content is solely the responsibility of the authors and does not necessarily represent the official views of the National Institutes of Health. Conflict of interest: There are no conflicts of interest. Authorship: S.N.: data analysis and manuscript drafting; M.T.: data analysis support and manuscript revising; L.R. and L.A.: conception and design of research and manuscript revising; E.F., B.L. and R.P.: interpretation of data and manuscript revising. All authors had responsibility for final content and read and approved the final manuscript. Ethics of human subject participation: This study was conducted according to the guidelines laid down in the Declaration of Helsinki, and all procedures involving research study participants were approved by the Battelle Memorial Institute Institutional Review Board. Written informed consent was obtained from all participants.

\section{Supplementary material}

For supplementary material accompanying this paper visit https://doi.org/10.1017/S1368980021002536

\section{References}

1. US Department of Agriculture \& Economic Research Service (2020) Key Statistics \& Graphics. US Department of 
Agriculture, Economic Research Service; available at https:// www.ers.usda.gov/topics/food-nutrition-assistance/food-secu rity-in-the-us/key-statistics-graphics.aspx (accessed April 2021).

2. Shankar P, Chung R \& Frank DA (2017) Association of food insecurity with children's behavioral, emotional, and academic outcomes: a systematic review. J Dev Behav Pediatr 38, 135-150.

3. Kimbro RT \& Denney JT (2015) Transitions into food insecurity associated with behavioral problems and worse overall health among children. Health Aff Proj Hope 34, 1949-1955.

4. Kral TVE, Chittams J \& Moore RH (2017) Relationship between food insecurity, child weight status, and parentreported child eating and snacking behaviors. I Spec Pediatr Nurs 22, e12177.

5. US Department of Health Human Services (2018) Physical Activity Guidelines for Americans, 2nd ed. US Department of Health Human Services; available at https://health.gov/ sites/default/files/2019-09/Physical_Activity_Guidelines_ 2nd_edition.pdf (accessed October 2019).

6. Hong I, Coker-Bolt P, Anderson KR et al. (2016) Relationship between physical activity and overweight and obesity in children: findings from the 2012 National Health and Nutrition Examination Survey National Youth Fitness Survey. Am J Occup Ther 70, 7005180060p1-7005180060p8.

7. Fram MS, Ritchie LD, Rosen N et al. (2015) Child experience of food insecurity is associated with child diet and physical activity. J Nutr 145, 499-504.

8. Asfour L, Natale R, Uhlhorn S et al. (2015) Ethnicity, household food security, and nutrition and activity patterns in families with preschool children. J Nutr Educ Behav 47, 498.e1-505.e1.

9. To QG, Frongillo EA, Gallegos D et al. (2014) Household food insecurity is associated with less physical activity among children and adults in the U.S. population. J Nutr 144, 1797-1802.

10. Ke J \& Ford-Jones EL (2015) Food insecurity and hunger: a review of the effects on children's health and behaviour. Paediatr Child Health 20, 89-91.

11. Food Resources and Action Centre (2017) The Impact of Poverty, Food Insecurity, and Poor Nutrition on Health and Well-Being. Food Resources and Action Centre; available at https://frac.org/wp-content/uploads/hunger-healthimpact-poverty-food-insecurity-health-well-being.pdf (accessed October 2019).

12. National Physical Activity Plan (2018) The 2018 United States Report Card on Physical Activity for Children and Youth. Washington, D.C.: National Physical Activity Plan.

13. Sagatov RDF, John LV, Gregoriou M et al. (2018) Recruitment outcomes, challenges and lessons learned: the Healthy Communities Study. Pediatr Obes 13, Suppl. 1, 27-35.

14. Strauss WJ, Nagaraja J, Landgraf AJ et al. (2018) The longitudinal relationship between community programmes and policies to prevent childhood obesity and BMI in children: the Healthy Communities Study. Pediatr Obes 13, 82-92.

15. John LV, Gregoriou M, Pate RR et al. (2015) Operational implementation of the healthy communities study: how communities shape children's health. Am J Prev Med 49, 631-635.

16. Bickel G, Nord M, Price C et al. (2000) Guide to Measuring Housebold Food Security, Revised 2000. Alexandria, VA: U.S. Department of Agriculture, Food and Nutrition Service.

17. Hager ER, Quigg AM, Black MM et al. (2010) Development and validity of a 2 -item screen to identify families at risk for food insecurity. Pediatrics 126, e26-e32.

18. Jyoti DF, Frongillo EA \& Jones SJ (2005) Food insecurity affects school children's academic performance, weight gain, and social skills. J Nutr 135, 2831-2839.
19. Pate RR, McIver K, Colabianchi N et al. (2015) Physical activity measures in the healthy communities study. $\mathrm{Am} \mathrm{J}$ Prev Med 49, 653-659.

20. van Buuren S \& Groothuis-Oudshoorn K (2011) MICE: multivariate imputation by chained equations in R. J Stat Softw 45, 1-67.

21. Tibshirani R (1996) Regression shrinkage and selection via the lasso. J R Stat Soc Ser B Methodol 58, 267-288.

22. Ritchie LD, Woodward-Lopez G, Au LE et al. (2018) Associations of community programs and policies with children's dietary intakes: the healthy communities study. Pediatr Obes 13, 14-26.

23. Pate RR, Frongillo EA, McIver KL et al. (2018) Associations between community programmes and policies and children's physical activity: the healthy communities study. Pediatr Obes 13, Suppl. 1, 72-81.

24. Burke MP, Frongillo EA, Jones SJ et al. (2016) Household food insecurity is associated with greater growth in body mass index among female children from kindergarten through eighth grade. J Hunger Environ Nutr 11, 227-241.

25. Au LE, Zhu SM, Nhan LA et al. (2019) Household food insecurity is associated with higher adiposity among US schoolchildren ages 10-15 years: the healthy communities study. J Nutr 149, 1642-1650.

26. Frongillo EA, Jyoti DF \& Jones SJ (2006) Food stamp program participation is associated with better academic learning among school children. J Nutr 136, 1077-1080.

27. Frongillo EA, Fram MS, Escobar-Alegría JL et al. (2019) Concordance and discordance of the knowledge, understanding, and description of children's experience of food insecurity among hispanic adults and children. Fam Community Health 42, 237-244.

28. Fram MS, Frongillo EA, Jones SJ et al. (2011) Children are aware of food insecurity and take responsibility for managing food resources. J Nutr 141, 1114-1119.

29. Centers for Disease Control and Prevention (2009) National Health and Nutrition Examination Survey Food Security Questionnaire 2009-2010. Centers for Disease Control and Prevention; available at https://www.cdc.gov/nchs/data/ nhanes/nhanes_09_10/fsq_f.pdf (accessed April 2020).

30. US Department of Agriculture \& Economic Research Services (2020) Survey Tools. US Department of Agriculture and Economic Research Services; available at https://www.ers. usda.gov/topics/food-nutrition-assistance/food-security-inthe-us/survey-tools/ (accessed April 2020).

31. Connell CL, Nord M, Lofton KL et al. (2004) Food security of older children can be assessed using a standardized survey instrument. J Nutr 134, 2566-2572.

32. Fram MS, Frongillo EA, Draper CL et al. (2013) Development and validation of a child report assessment of child food insecurity and comparison to parent report assessment.J Hunger Environ Nutr 8, 128-145.

33. Fram MS, Bernal J \& Frongillo EA (2015) The Measurement of Food Insecurity among Children: Review of Literature and Concept Note. Florence: UNICEF Office of Research.

34. Plimier CC, Hewawitharana SC, Webb KL et al. (2020) Community-level obesity prevention is not associated with dieting behaviors and weight dissatisfaction in children: the healthy communities study. Pediatr Obes 15, e12594.

35. Coleman-Jensen A, Gregory CA \& Singh A (2014) Household Food Security in the United States in 2013. Washington, D.C.: U.S. Department of Agriculture, Economic Research Service.

36. Coleman-Jensen A, Rabbitt MP, Gregory CA et al. (2016) Household Food Security in the United States in 2015. Washington, D.C.: U.S. Department of Agriculture, Economic Research Service. 
37. Fryar CD, Carroll MD \& Afful J (2020) Prevalence of Overweight, Obesity, and Severe Obesity among Children. Hyattsville, MD: National Center for Health Statistics.

38. Strauss WJ, Sroka CJ, Frongillo EA et al. (2015) Statistical design features of the healthy communities study. $A m \mathrm{~J}$ Prev Med 49, 624-630.

39. Hake M, Dewey A, Engelhard E et al. (2021) The Impact of the Coronavirus on Food Insecurity in 2020 \& 2021. Chicago, IL: Feeding America.
40. Coleman-Jensen A, Rabbitt MP, Gregory CA et al. (2020) Household Foods Security in the United States in 2019. Washington, D.C.: U.S. Department of Agriculture, Economic Research Service.

41. Ogden CL, Carroll MD, Fakhouri TH et al. (2018) Prevalence of obesity among youths by household income and education level of head of household - United States 2011-2014. MMWR Morb Mortal Wkly Rep 67, 186-189. 